\title{
An intestinal stem cell niche in Apc mutated neoplasia targetable by CtBP inhibition
}

\author{
Ayesha T. Chawla ${ }^{1}$, Agnes D. Cororaton ${ }^{2}$, Michael O. Idowu ${ }^{3,4}$, Priyadarshan K. Damle ${ }^{2}$, \\ Barbara Szomju2, Keith C. Ellis ${ }^{4,6}$, Bhaumik B. Patel ${ }^{2,4,5}$ and Steven R. Grossman ${ }^{2,4}$ \\ ${ }^{1}$ VCU Wright Center for Clinical and Translational Research, Virginia Commonwealth University, Richmond, VA 23298, USA \\ ${ }^{2}$ Department of Internal Medicine, Virginia Commonwealth University, Richmond, VA 23298, USA \\ ${ }^{3}$ Department of Pathology, Virginia Commonwealth University, Richmond, VA 23298, USA \\ ${ }^{4}$ VCU Massey Cancer Center, Virginia Commonwealth University, Richmond, VA 23298, USA \\ ${ }^{5}$ Department of Medicine, Hunter Holmes McGuire VA Medical Center, Richmond, VA 23249, USA \\ ${ }^{6}$ Department of Medicinal Chemistry, Virginia Commonwealth University, Richmond, VA 23298, USA \\ Correspondence to: Steven R. Grossman, email: steven.grossman@vcuhealth.org \\ Keywords: c terminal binding protein; adenomatous polyposis; tumor initiating cells \\ Received: May 19, $2018 \quad$ Accepted: June 19, $2018 \quad$ Published: August 21, 2018 \\ Copyright: Chawla et al. This is an open-access article distributed under the terms of the Creative Commons Attribution License \\ 3.0 (CC BY 3.0), which permits unrestricted use, distribution, and reproduction in any medium, provided the original author and \\ source are credited.
}

\section{ABSTRACT}

C-terminal binding protein 2 (CtBP2) drives intestinal polyposis in the Apc ${ }^{\text {min }}$ mouse model of human Familial Adenomatous Polyposis. As CtBP2 is targetable by an inhibitor of its dehydrogenase domain, understanding CtBP2's role in adenoma formation is necessary to optimize CtBP-targeted therapies in Apc mutated human neoplasia. Tumor initiating cell (TIC) populations were substantially decreased in Apc $^{\min }$ Ctbp2 $^{+/-}$intestinal epithelia. Moreover, normally nuclear Ctbp2 was mislocalized to the cytoplasm of intestinal crypt stem cells in Ctbp2 ${ }^{+/-}$mice, both Apc min and wildtype, correlating with low/absent CD133 expression in those cells, and possibly explaining the lower burden of polyps in Apc ${ }^{\min } \mathrm{Ctbp2}^{+/-}$mice. The CtBP inhibitor 4-chloro-hydroxyimino phenylpyruvate (4-CI-HIPP) also robustly downregulated TIC populations and significantly decreased intestinal polyposis in Apc ${ }^{\min }$ mice. We have therefore demonstrated a critical link between polyposis, intestinal TIC's and Ctbp2 gene dosage or activity, supporting continued efforts targeting CtBP in the treatment or prevention of Apc mutated neoplasia.

\section{INTRODUCTION}

Sporadic colon cancer is frequently characterized by mutation of the $A P C$ tumor suppressor, while autosomal dominant inheritance of a mutant $A P C$ allele, as in Familial Adenomatous Polyposis (FAP), results in early onset massive colonic polyposis that uniformly progresses to colorectal cancer unless prophylactic total colectomy is performed [1]. Recently, we have shown that the emerging oncogene and drug target, $\mathrm{C}$-terminal binding protein 2 (CtBP2), is a key driver of neoplasia in the $\mathrm{Apc}^{\mathrm{min} /+}(\mathrm{Apc}$ $\left.{ }^{m i n}\right)$ mouse model of human FAP [2].
C-terminal binding proteins 1 and 2 (CtBP) are paralogous transcriptional co-regulators frequently overexpressed and associated with worse outcome and aggressive tumor features in colon, [3] breast [4, 5], gastric [6], ovarian [7], and prostate [8] cancers. Indeed, the majority of colon and breast tumors demonstrate overexpression of CtBP1 and/or CtBP2 proteins (64\% [3] and $92 \%$ [4], respectively). CtBP upregulation leads to inappropriate cell survival as well as enhanced migratory and invasive properties due to the ability of CtBP to repress transcription of Bik [9], Brcal [4], PTEN [10], the epithelial adhesion protein E-cadherin [11], and many other tumor suppressive genes $[11,12]$, as well as co- 
activate the migration-associated gene Tiam1 [13] and the drug efflux pump MDR1 [14]. In vitro overexpression of CtBP is oncogenic in a manner similar to mutant H-Ras [2], transforming primary mouse embryo fibroblasts to anchorage-independent growth, which is a strong predictor of tumor growth in mouse xenograft models [15]. Importantly, CtBP transcriptional co-regulation is activated by an increase in NADH concentration, as is often the case in hypoxic and/or glycolytically active tumors [16], due to NADH-dependent oligomerization of CtBP's conserved dehydrogenase domain [17].

The functional dehydrogenase domain encoded by $\mathrm{CtBP} 1 / 2$ is targetable by small molecule analogues of its native substrate $\alpha$-keto- $\gamma$-(methylthio) butyric acid (MTOB). Of these analogues, 2-hydroxyimino-3-phenylpropionic acid (HIPP) and its more potent 4-chloroderivative (4-Cl-HIPP), antagonize CtBP's proposed oncogenic functions [18]. Pharmacological inhibition of $\mathrm{CtBP}$ using HIPP profoundly reduced intestinal polyposis in $A p c^{\text {min }}$ mice, similarly to haploinsufficiency of $C t b p 2$ [2], and with no observable toxicity.

CtBP2's role in driving a tumor initiating cell (TIC) niche in solid tumors is emerging [19]. TICs contribute to intra-tumoral heterogeneity, metastasis and chemoresistance in a variety of solid cancers, including colon, pancreatic and ovarian, among other cancers [2024], and an ideal therapy would target this population to overcome metastatic or local relapse from treatmentresistant TICs. Both normal stem cell and TIC populations from intestinal epithelia exhibit the same cell surface markers, though underlying molecular events, such as $A P C$ allelic loss, transform normal intestinal stem cells into TIC's [21, 25]. A number of TIC-related cell surface markers have been identified in the intestine, including CD44, CD24, CD133, and CXCR4 (23, 28-32). CD44+/ CD24+ populations obtained from colorectal tumors can initiate growth of colonospheres in vitro and colorectal tumors in vivo, and similar cells exhibit TIC activity across a variety of solid tumors [26]. The progeny of CD133+ cells are capable of giving rise to all major intestinal cell types, as well as neoplasms [21, 27], and CD133+ marks long-lived multipotent intestinal stem cells [28]. CXCR4, a chemokine receptor, is expressed both in normal tissue stem cells of the breast, lung, and prostate gland, as well as tumors formed in those organs [29-32] Consistent with a role of colon TIC's, colon cancer micrometastases require CXCR4 to initiate proliferation, and CXCR4/CD133 dual positive cells demonstrate enhanced tumorigenic capabilities over unsorted cells $[33,34]$. CXCR4 is also found to be overexpressed in the majority of colon cancer cases $[35,36]$.

In this work we have interrogated the effect of Ctbp2 haploinsufficiency on the intestinal stem cell niche of both wild type and $A p c^{\text {min }}$ mice, to better understand Ctbp2's biologic role in the expanded stem cell population in $A p c^{\text {min }}$ mice that serve as precursors to polyps, which are enriched for cells with stem cell-like markers that are characterized as tumor initiating cells (TIC's). We show that Ctbp2 loss or inhibition with 4-Cl-HIPP both reduce normal stem cell and TIC populations in $A p c^{\text {min }}$ intestine, with a surprising finding of $\mathrm{Ctbp} 2$ protein misolocalization to the cytoplasm of stem cells and TIC's when haploinsufficient. This mislocalization could explain the profound effect of Ctbp2 haploinsufficiency on polyp number and survival in $A p c^{\text {min }}$ mice and supports further therapeutic development of $\mathrm{CtBP}$ as a target in $A P C$ mutated neoplasia.

\section{RESULTS}

\section{Ctbp2 gene dosage determines TIC abundance in $A p c^{\text {min }}$ intestine}

To understand the role of Ctbp2 specifically in TIC activity induced by $A p c$ mutated neoplasia, we first compared CD44+/CD24+ as well as CD133+/CXCR4+ populations (which include both normal stem cells and TIC's) in small intestinal epithelia obtained from $A p c^{\mathrm{min} /+}$, $C t b p 2^{+/-} A p c^{m i n /+}$, wildtype and Ctbp $2^{+/-}$mice (Figure 1A, Supplementary Figure 1A). Dual positive CD44+/CD24+ and CD133+/CXCR4+ cells were at least 2-fold less

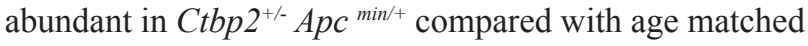
$A p c^{\mathrm{min} /+}$ epithelia (Figure 1B, Supplementary Figure 1B). CD24+/CD44+ and CD133+/ CXCR4+ normal stem cell populations were also decreased 2-fold in non-neoplastic $C t b p 2^{+/}$compared with wildtype intestinal epithelia (Figure 1A), indicating control of Ctbp2 over both normal and neoplastic (TIC) stem cell populations.

Histologic examination of CD133 expression by immunofluorescence in age matched $A p c^{\min /+}$ vs. Ctbp2 ${ }^{+/} A p c^{\text {min/+ }}$ mouse small intestinal polyps (Figure 1C-1D) suggested that CD133 expression was abundant, although not consistent, throughout the adenomatous polyps of Apc ${ }^{\mathrm{min} /+}$ mice, as has been reported [27] (Figure 1C). Interestingly, the few adenomatous polyps from $C t b p 2^{+/}$ $A p c^{\mathrm{min} /+}$ mice exhibited significantly diminished CD133+ expression (Figure 1D-1E), along with markedly less proliferative potential, as determined by Ki-67 staining, towards the edge of the polyp (Supplementary Figure 1C), as compared with $A p c^{\text {min/+ }}$ adenomas.

Expression of CD133 in normal crypts of aforementioned genotypes revealed that CD133 was equivalently expressed throughout the lower half of the intestinal crypts of $A p c$ wildtype and $A p c^{\text {min }}$ small intestines (See empty arrows, CD133 panel, Figure 2A$2 \mathrm{D}, 2 \mathrm{E})$, especially in transit amplifying cells and early progenitors, as previously reported [27].

Consistent with Ctbp2's nuclear role in coregulation of Wnt target genes [37], Ctbp2 expression in Apc min (See arrows, DAPI and Ctbp2 panels, Figure 2A) and $A p c$ wildtype (See arrows, DAPI and Ctbp2 panels, Figure 2B) normal crypt intestinal stem cells, or the "stem cell zone" 
(See circled area indicating stem cell zone in Merge panel, Figure 2A), was robust and predominantly nuclear (See arrows indicating nuclear Ctbp2 in Merge panel, Figure 2A). Staining for Ctbp2 in normal intestinal crypts from Ctbp2 haploinsufficient genotypes (Apc ${ }^{\text {min }}$ or $A p c W T$ ) demonstrated markedly less nuclear Ctbp2 expression and mostly cytoplasmic expression in the stem cell zone (See arrows, DAPI and Ctbp2 panels Figure 2C and 2D). Moreover, the stem cell zone of Ctbp2 haploinsufficient crypts where cytoplasmic Ctbp2 was observed, also showed partial loss of CD133 expression (See empty arrows in CD133 panel and circles in Merge panel, Figure $2 \mathrm{C}$ and 2D), with the average number of CD133+ cells in $C t b p 2^{+/-}$crypts significantly lower than in $C t b p 2^{+/+}$crypts (Figure 2E). This indicates a tight correlation between stemness (i.e. CD133 expression) and Ctbp2 nuclear localization, suggesting that the decrease in polyps in $C t b p 2^{+/-} A p c^{\min /+}$ mice [2] and the lower abundance of stem cells in Ctbp2 haploinsufficient polyps (Figure 1D$1 \mathrm{E})$, may, in part, be due to cytoplasmic mislocalization of Ctbp2 that correlates with loss of crypt stemness (Figure 2C-2D).

The expression of CD133 was otherwise intact in the stem cell zone of intestinal crypts from Ctbp2 WT mice (both $A p c^{\text {min }}$ and $A p c$ wildtype), suggesting that both alleles of $C t b p 2$ must be present to sustain expression of CD133 in the stem cell zone. By inference, there is a need for a full complement of Ctbp2 to maintain a phenotypically normal crypt stem cell compartment. Also consistent with this finding in normal crypts, Ctbp2 was predominantly cytoplasmic in adenomatous cells from $C t b p 2^{+/-} A_{p c} c^{m i n /+}$ small intestinal polyps as compared with its usual nuclear expression in $C t b p 2^{+/+} A p c^{m i n /+}$ polyps (Figure 3A-3B), suggesting the mechanism that led to Ctbp2 cytoplasmic localization in normal crypt stem cells, carried forward when stem cells transformed to form TIC's and adenomatous polyps.

\section{Pharmacological inhibition of Ctbp limits polyposis and intestinal stem cell/TIC abundance in $A p c^{\text {min }}$ mice}

As we have identified Ctbp2 as a key dependency for both $A p c^{\text {min }}$ intestinal polyposis and TIC populations, we investigated whether CtBP chemical inhibition, which can also suppress polyposis, likewise reduced TIC populations. The $1^{\text {st }}$ generation CtBP inhibitor, HIPP, targets the dehydrogenase domain of $\mathrm{CtBP}$, diminishes CtBP function [2], and suppresses polyp formation by $50 \%$ in $A p c^{\text {min }}$ mice [2]. Given the high dose of HIPP required in
A
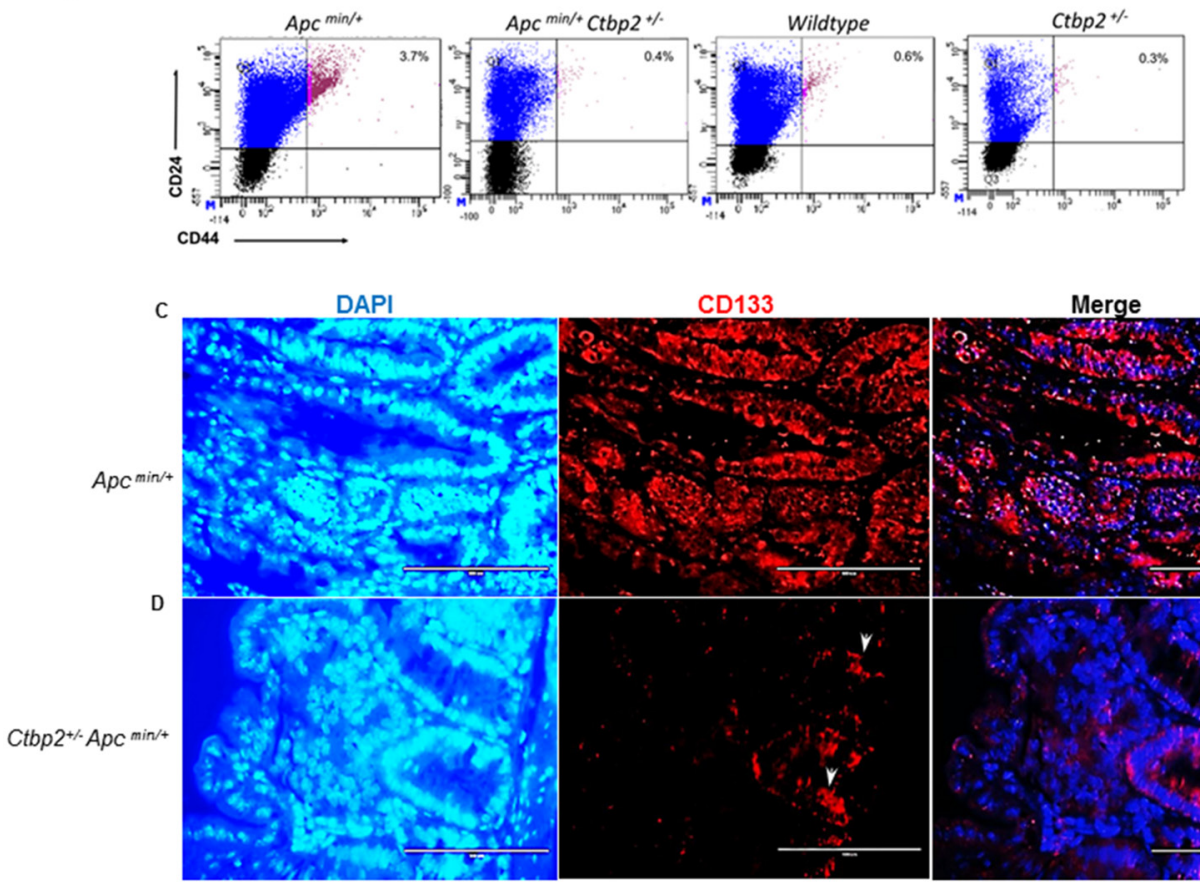
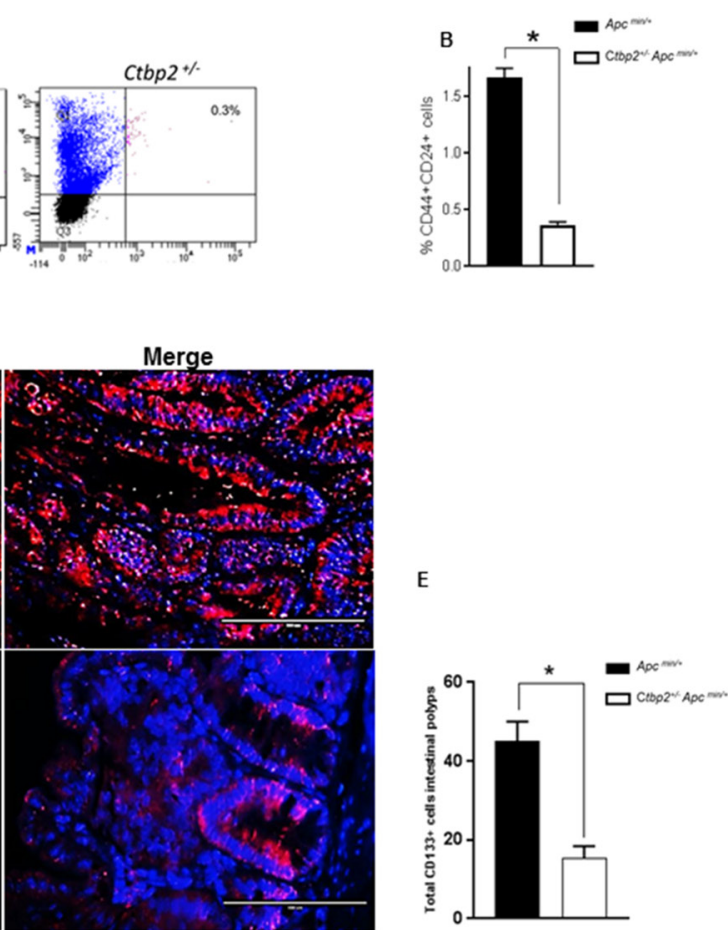

Figure 1: Ctbp2 haploinsufficiency decreases TIC populations in $A p c^{m i n /+}$ intestinal epithelia. (A) Scatter plots of representative flow cytometric analyses of intestinal epithelial cells for CD44 and CD24 with top right quadrants representing percentage of CD44+CD24+ cells in age-matched mice of indicated genotypes. (B) Quantification of CD44+CD24+ cells from intestinal epithelia of indicated genotypes; $n=3$ biologic replicates. ${ }^{*} \mathrm{p}<0.05$ for all analyses, error bars represent standard deviation from the mean. (C, D) IF staining for CD133+ cells on paraffin sections of intestinal polyps from age-matched (4 months) mice of indicated genotypes using antiCD133 antibodies followed by Alexa flour 594 secondary antibody and DAPI stain (blue) to define nuclei; representative CD133 positive cells in Ctbp2 heterozygous polyps indicated by arrows. (E) Total CD133 positive cells in mouse small intestine obtained from indicated genotypes ( $\mathrm{n}=3$ polyps/ mouse, ${ }^{*} \mathrm{p}<0.05$, error bars represent standard deviation from the mean). 
vivo, we tested the most potent available HIPP derivative, 4-Cl-HIPP [18], for suppression of polyposis and effect on TIC populations in $A p c^{\text {min }}$ mice (Supplementary Figure 2). Previous studies performed with 4-Cl-HIPP suggest that it is a CtBP2 specific inhibitor due to its on-target ability to abrogate $\mathrm{CtBP} 2$-mediated transcriptional repression [18]. Using dosing with minimal toxicity to major organs (Supplementary Figure 2A), we saw that 4-Cl-HIPP was as effective as HIPP at suppressing polyposis at 8 weeks, with a $60 \%$ reduction noted, and at a dose $60 \%$ lower than HIPP. (Figure 4A, Supplementary Figure 2B-2C). Similar to the effect of Ctbp2 haploinsufficiency, we observed a significant 5-fold decline in the percentage of dual positive CD44+CD24+ cells in intestinal epithelial cells of $A p c^{\text {min }}$ mice receiving 4-Cl-HIPP as compared to vehicle (Figure 4B-4C).

\section{Pharmacological inhibition of Ctbp blocks intestinal Wnt signalling}

Colorectal cancers often exhibit aberrant activation of the Wnt/ $\beta$ catenin pathway leading to activation of downstream oncogenic targets, such as cyclin D1, c-Myc and the intestinal stem cell regulatory gene, Lgr5 $[21,25]$. Since Ctbp2 plays a key role in Wnt/ßcatenin mediated signaling by coactivating TCF4 [37], we sought to assess if pharmacological inhibition of Ctbp2 using 4-Cl-HIPP inhibits oncogenic Wnt signaling in $A p c^{\text {min }}$ intestinal epithelia, as we had previously found in Ctbp2 haploinsufficient mice [2]. Indeed, the mRNA expression of c-Myc and Lgr5 was suppressed in 4-ClHIPP vs. vehicle-treated $A p c^{\text {min }}$ intestinal cells (Figure 4D). 4-Cl-HIPP was also able to suppress Ctbp2 mRNA

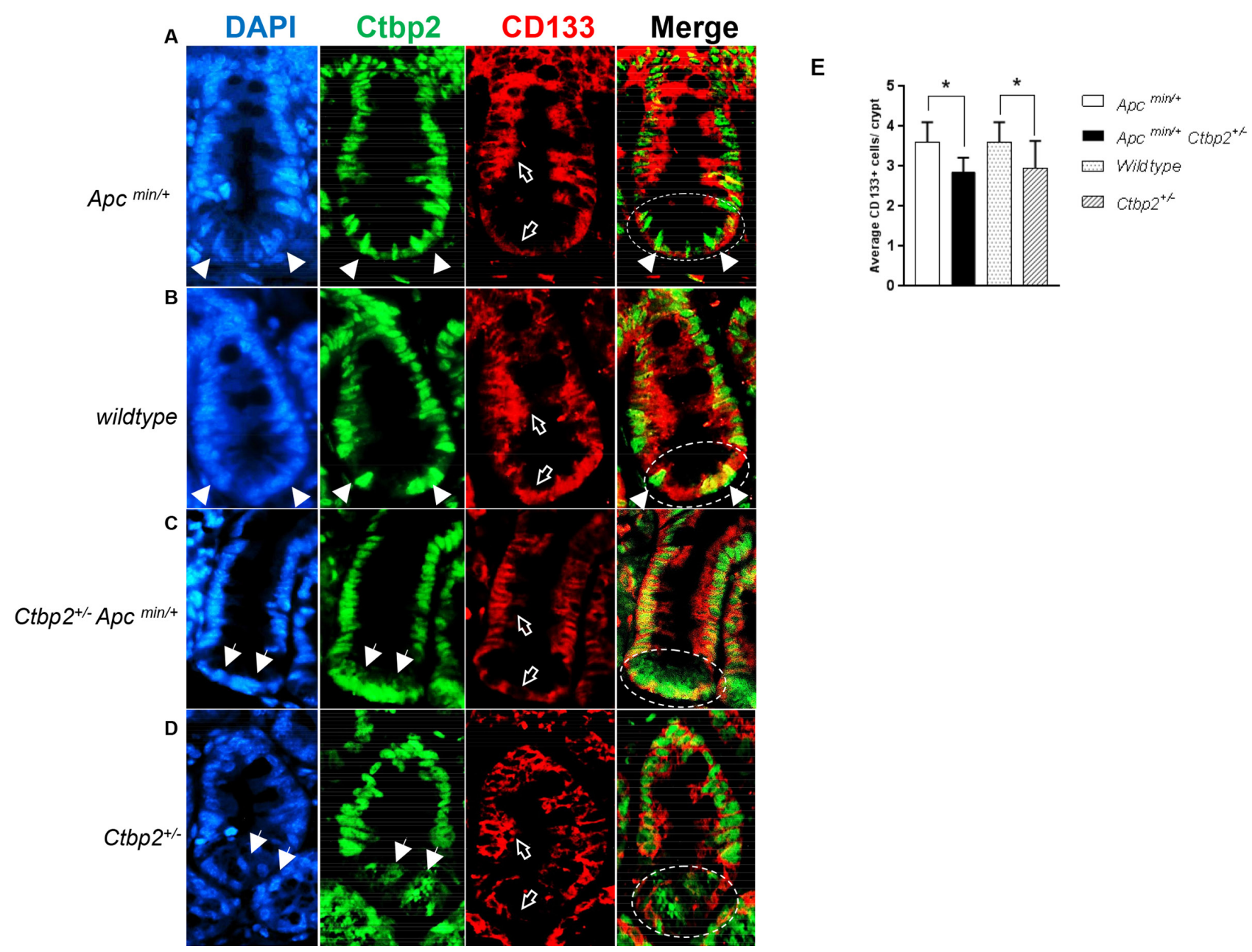

Figure 2: CD133 expression is ablated in Ctbp2 haploinsufficient intestinal crypts. (A-D) IF staining of normal small intestinal crypts for CD133 (red, empty arrows) and Ctbp2 (green, filled arrows) using anti-CD133 and anti-Ctbp2 antibodies followed by Alexa flour 594 and Cruz flour 488 secondary antibodies on paraffin sections from age-matched (4 months) mice of indicated genotypes. DAPI stain (blue) defines nuclei. The stem cell zone is represented in circles in the Merge view. Magnification $=400 X$. (E) Average CD133 positive cells per small intestinal crypt from each genotype in (A-D) determined by counting CD133+ cells from 2 mice of each genotype and 10 crypts per mouse intestine, ${ }^{*}$ p value $<0.005$, error bars represent standard deviation from the mean. 


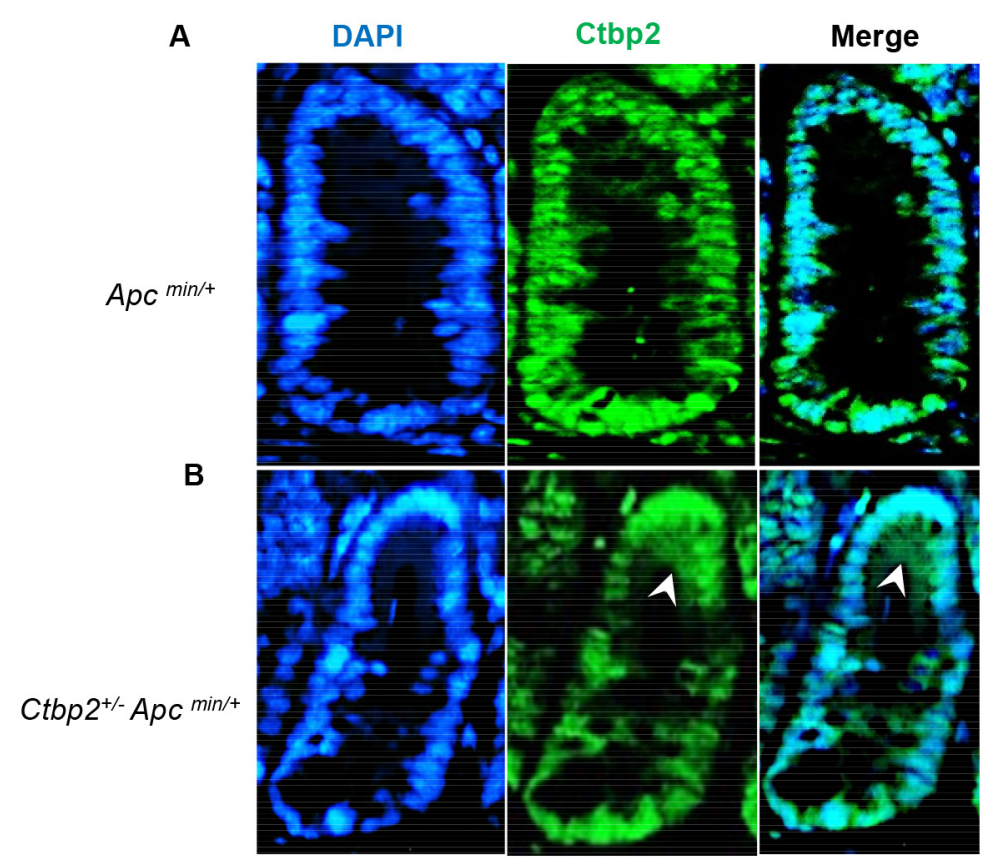

Figure 3: Ctbp2 localization in Ctbp2 wildtype vs. Ctbp2 haploinsufficient $A_{p c}{ }^{\text {min }}$ small intestinal polyps. Ctbp2 expression (green) was determined by IF with anti-Ctbp2 antibody and Cruz flour 488-conjugated secondary antibody in small intestinal polyps from age-matched (4 months) (A) $A p c^{\mathrm{min} /+}$ and (B) $C t b p 2^{+-} A p c^{\mathrm{min} /+}$ mice. Arrows indicate cells with cytoplasmic Ctbp2 localization in (B). DAPI (blue) stain indicates nuclei. Magnification $=400 \mathrm{X}$.
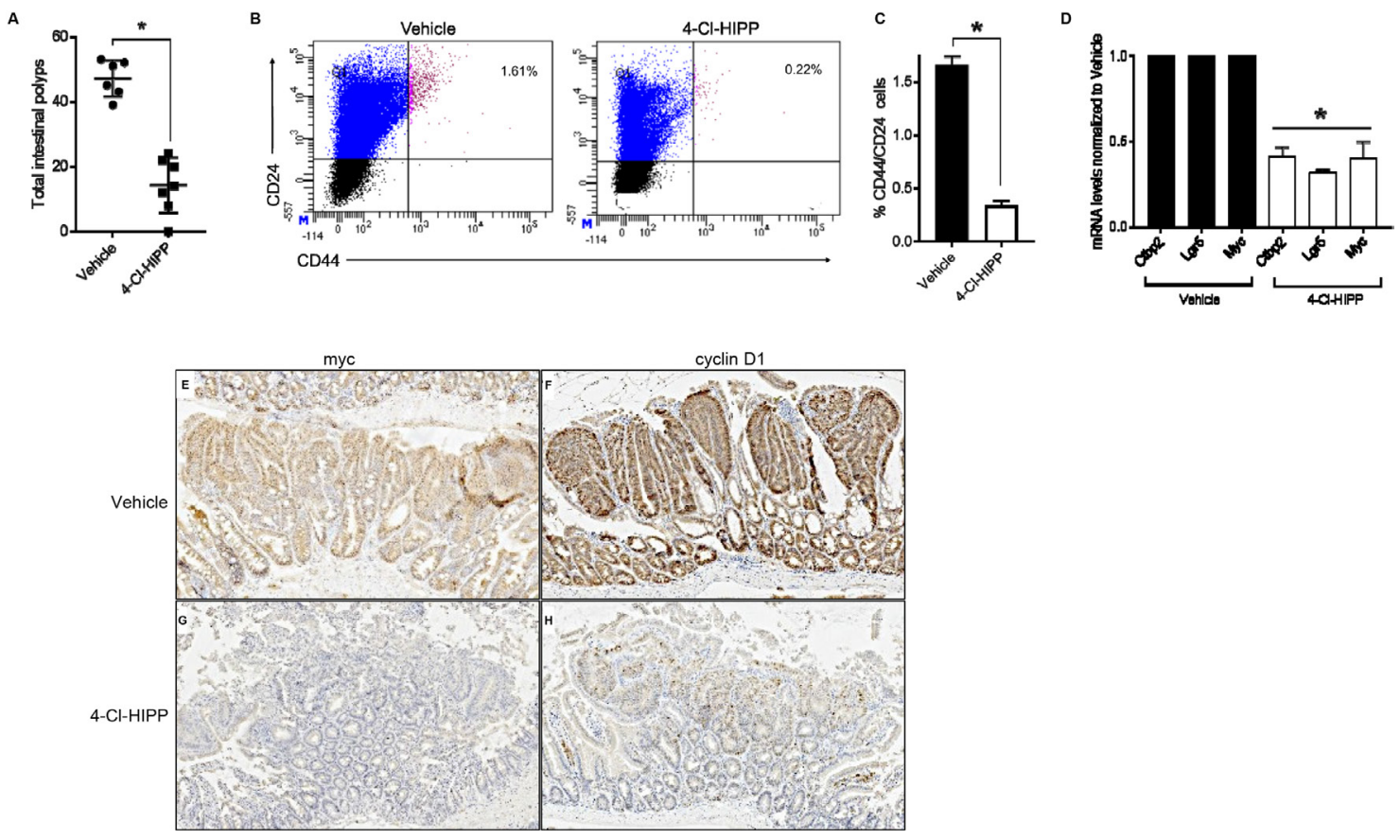

Figure 4: Pharmacological inhibition of Ctbp2 decreases polyposis and TIC populations in $A p c^{\text {min/+ }}$ intestinal epithelia. (A) Quantification of total intestinal polyps from Vehicle $(\mathrm{n}=6)$ or 4-Cl-HIPP $(\mathrm{n}=7)$ treated $A p c^{\min /+}$ mice after 8 weeks of 4-Cl-HIPP treatment, $\mathrm{p}<0.02$, error bars indicate $1 \mathrm{SD}$. (B) Scatter plot of representative flow cytometric analysis of intestinal epithelial cells with top right quadrants representing percentage of CD44+CD24+ cells in age matched mice treated with vehicle or 4-Cl-HIPP for 8 weeks. (C) Quantification of CD44+CD24+ population of cells in (B) ( $\mathrm{n}=4$ biologic replicates); ${ }^{*} \mathrm{p}<0.02$, error bars represent standard deviation from the mean. (D) mRNA levels of TIC-associated genes expressed in intestinal epithelial cells from vehicle or 4-Cl-HIPP treated mice; bars represent mean values from amplification of mRNA from intestinal epithelial cells obtained from 3 mice per group; ${ }^{*} \mathrm{p}<0.05$, error bars represent standard deviation from the mean. (E-H) IHC staining of $A p c^{m i n / t}$ intestinal polyps from mice treated with vehicle or 4-Cl-HIPP, for c-Myc (E, G) or cyclin D1 (F, H). 
levels, possibly reflecting transcriptional autoregulation of the Ctbp 2 gene by Ctbp2. The qPCR data was further supported by IHC of 4-Cl-HIPP vs. vehicle-treated Apc ${ }^{m i n}$ small intestine, which revealed decreased c-Myc and cyclin D1 staining in polyps (Figure 4E-4H; stain intensity $3+$ (vehicle) and $1+(4-\mathrm{Cl}-\mathrm{HIPP})$ for c-Myc and cyclin D1). Pharmacological inhibition and genetic ablation of Ctbp2 are therefore both associated with reduction in polyposis, diminished intestinal TIC populations, and reduction in Wnt pathway oncogenic signaling in $A p c^{\text {min }}$ intestine. Moreover, the concordance of genetic and pharmacologic data support 4-Cl-HIPP's activity as reflecting on-target antagonism of $\mathrm{Ctbp}$.

\section{CtBP2 ablation or pharmacologic inhibition attenuates TIC function and Wnt signalling in human colon cancer cells}

We have illustrated efficient 4-Cl-HIPP inhibition of intestinal TIC populations in the $A p c^{\text {min }}$ polyposis (precancer) model. To determine if 4-Cl-HIPP could similarly inhibit human TIC populations in cancer cells, and mirror effects of CtBP2 deficiency, we utilized human colorectal cancer cell lines that form tumor spheroids (tumorspheres) that are enriched for TIC's [19] to assay the effect of CtBP inhibition or CtBP2 genetic depletion. We generated HCT116 colon cancer cells genetically deficient for $C t B P 2$ using CRISPR knockout techniques (Figure 5A, 5D), and compared the effect of 4-Cl-HIPP treatment vs. $C t B P 2$ knockout on tumorsphere growth. Notably, 4-Cl-HIPP efficiently disrupted primary sphere formation of HCT116 cells (Figure 5B). Additionally, HCT116 cells with CRISPR-mediated deletion of both $C t B P 2$ alleles were also unable to form primary tumorspheres (Figure 5C), suggesting tumorsphere inhibition, and by inference, TIC inhibition, is an ontarget activity of 4-Cl-HIPP.

Translating our TIC marker and Wnt pathway results from $A p c^{\text {min }}$ mouse intestinal epithelia to human colon cancer cells, and consistent with CtBP's regulation of TIC-enriched tumorspheres, LGR5 mRNA and/ or protein expression was also effectively disrupted
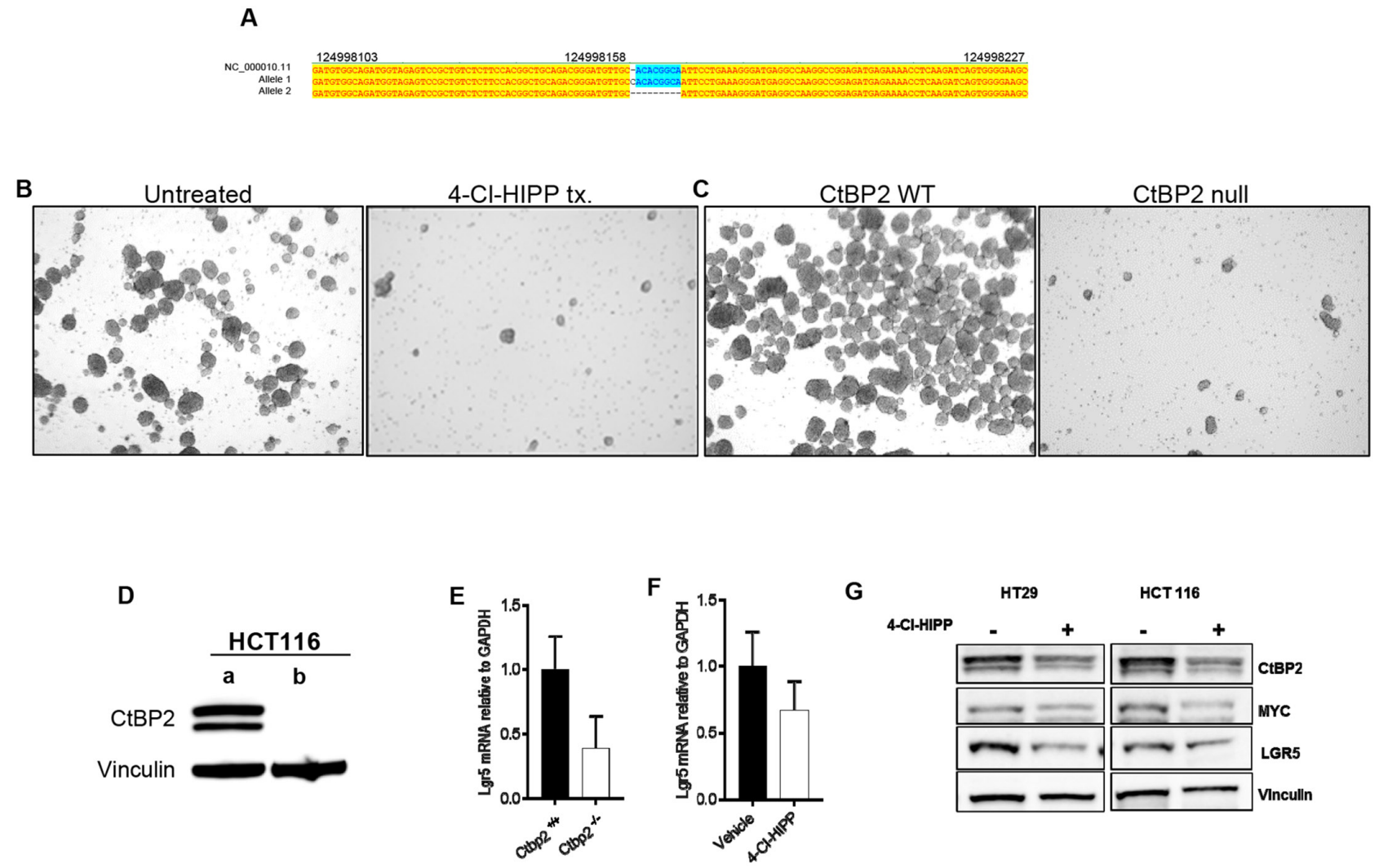

Figure 5: CtBP2 null human colon cancer cells have decreased TIC expression. (A) ClustalW alignment of sequences of the HCT116 CtBP2 CRISPR knockout clone. Subcloned colonies from CRISPR targeting were sequenced using universal primer M13 F43, and representative clones aligned with wildtype $C t B P 2$ sequence. One allele has an insertion of one base pair while the other allele has a deletion of eight base pairs (B) HCT116 primary tumorspheres treated with vehicle or 4-Cl-HIPP after 5 days of incubation. (C) HCT116 tumorspheres prepared from parental (left) or CtBP2 null clone (right) after 5 days in culture. (D) Immunoblot analysis of CTBP2 expression in HCT116 (a) parental cells, and (b) CTBP2 null sub-clone from (A). Vinculin was a loading control. (E, F) Relative Lgr5 mRNA levels in (E) HCT116 parental vs. CtBP2-null tumorspheres or (F) HCT116 tumorspheres treated with vehicle or 4-Cl-HIPP. (G) Immunoblot of vehicle vs. 4-Cl-HIPP treated HT29 and HCT116 tumorsphere lysates using indicated antibodies. 
by $C t B P 2$ knockout in HCT116 tumorspheres (Figure $5 \mathrm{E}$ ), or by 4-Cl-HIPP treatment in HCT116 or HT29 tumorspheres (Figure 5F-5G). Along with LGR5 regulation, we have demonstrated that $C t b p 2$ deficiency is associated with downregulation of Wnt pathway oncogenic effectors [2] and 4-Cl-HIPP likewise inhibited downstream Wnt pathway targets c-Myc and cyclin D1 in $A p c^{\text {min }}$ polyps (Figure 4E-4H). We therefore examined the effect of 4-Cl-HIPP on c-Myc expression in HCT116 tumorspheres, as c-Myc is a downstream oncogenic target of the Wnt/ TCF4 pathway [38], and observed a robust decrease in c-Myc protein levels after 4-Cl-HIPP treatment (Figure 5G). Thus, 4-Cl-HIPP can phenocopy CtBP2 knockout and inhibit the Wnt target and TIC marker LGR5, and also attenuate expression of the key oncogenic Wnt pathway target c-Myc in human colon cancer cells.

\section{DISCUSSION}

Years of study in cell culture and in human tumors have implicated CtBP1 and 2 as oncogenic, by virtue of observed activities in TIC regulation, EMT induction, chemoresistance and common overexpression in solid tumors linked to poor outcome [39]. Our recent observations that CtBP2 is a key dependency in Apcmutated neoplasia, was the first in vivo evidence of CtBP2's neoplastic potential [2]. In this work, we have determined the biologic basis of CtBP2's neoplastic activities in $A p c$-mutated neoplasia as linked to its regulation of TIC populations and transcriptional regulation of downstream Wnt targets, such as LGR5, c-Myc and cyclin D1. Moreover, relative deficiency of Ctbp2, or functional inhibition, in the early progenitor niche in crypts, may suppress $A p c$-deficiency associated neoplasia, as Ctbp2 is a key component of the pathway between $A p c$ loss and neoplastic transformation, via its regulation of c-Myc and cyclin D1.

The surprising findings relative to Ctbp2's unique cytoplasmic localization when genetically haploinsufficient, could explain the drastically lower TIC and polyp count in $\mathrm{Ctbp}^{+/-} \mathrm{Apc}^{\mathrm{min} /+}$ mice, as Ctbp2 localized in the cytoplasm would be partially or fully inactive to perform transcriptional coregulation of Wnt target genes [40]. As a result, crucial Wnt targets that play a driver role in cell proliferation and TIC function, such as c-Myc and cyclin D, would be expressed at lower levels, as already observed in Ctbp2 haploinsufficient $A p c^{\text {min }}$ polyps [2], and thus render stem cell precursors to TIC's less likely to transform. Cytoplasmic accumulation of Ctbp 2 in the stem cell zone of normal (non-adenomatous) crypts, along with partial loss of CD133 in the setting of Ctbp2 haploinsufficiency, is evidently compatible with normal intestinal development, but could have implications in slower recovery of intestinal crypt regeneration and higher sensitivity of the gut to stress or injury, as the stem cell reserve may be limited. Still unclear, is exactly why Ctbp2 is found in the cytoplasm when it is haploinsufficient, which requires further study, but may be related to stoichiometry of its binding to APC protein (which like Ctbp2, also exhibits a dot like pattern in the cytoplasm [41]). Thus, Ctbp2 is potentially a key driver of transformation of normal crypt CD133+ stem cells to adenomatous polyps, and thus, a driver of the intestinal TIC phenotype in $A p c$ mutated intestinal neoplasia.

Our evaluation of the $2^{\text {nd }}$ generation CtBP inhibitor 4-Cl-HIPP demonstrates that targeting CtBP is safe and efficacious in the $A p c^{\text {min }}$ model, and moreover, its effect on TIC populations in vivo and in vitro phenocopies Ctbp 2 knockout, consistent with on-target activity. Further evaluation of 4-Cl-HIPP and related inhibitors, alone or in combination with standard therapies, is warranted in colorectal cancer. Our data also suggests that anti-CtBP therapy, in general, may serve as a novel anti-TIC therapy in settings where TIC's represent a mechanism for cancer relapse and/or chemoresistance [42].

\section{MATERIALS AND METHODS}

\section{Cell culture and sphere assay}

HCT116 cells and HT29 cells (ATCC) were maintained in RPMI 1640 medium in tissue culture treated plates and passaged using trypsin-EDTA upon $70 \%$ confluency. For tumorsphere assays, stem cell media (SCM) was prepared as follows; DMEM/ F12 media supplemented with $1 \%$ penicillin/ streptomycin, $20 \mathrm{ng} / \mathrm{ml}$ epidermal growth factor, $10 \mathrm{ng} / \mathrm{ml}$ fibroblast growth factor and B27 were used. Tumorsphere culture was maintained using 200 cells/ well of an ultra-low attachment plate in SCM and measured for protein and mRNA at the end of 5 days. For primary tumorspheres, cells were seeded in SCM on day one and passaged on day 5 for secondary tumorspheres. On day 10, tumorspheres were passaged for tertiary spheres and harvested on day 15 .

\section{Western blotting}

Cells were washed in cold PBS and lysed in RIPA buffer $(150 \mathrm{mM} \mathrm{NaCl}, 50 \mathrm{mM}$ Tris HCL, pH 8.0, 1.7\% NP-40, 0.17\% sodium dodecyl sulfate (SDS), 0.5\% Nadeoxycholate monohydrate, 5mM EDTA) containing 1 tablet of complete Mini Protease Inhibitor Cocktail /10ml (Roche). Lysates were cleared of insoluble material by centrifugation at 15,000 RPM. Proteins (30 $\mu \mathrm{g})$ were loaded onto a Bis-Tris 4-12\% gel containing NuPAGE MOPS buffer. Antibodies- Ctbp2 (Cat no. 612044, BD transduction); c-Myc (N-262; Cat no. sc-764, Santa Cruz).

\section{Real-time polymerase chain reaction (qPCR) analysis}

Total RNA was isolated using RNeasy (Qiagen) and manufacturer's protocol was followed. $1 \mu \mathrm{g}$ total 
RNA was reverse transcribed using sensiFAST cDNA (Bioline). The cDNA was amplified in triplicate using SYBR green and the specific primers were used to detect amplicons. The primers used for qPCR were as follows human CtBP2 F 5'- ATCCACGAGAAGGTTCTAAACGA -3', R- 5'CCGCACGATCACTCTCAGG -3'; human LGR5 F- 5'- GGAGGAGGGAGAACCCACTT -3', R- 5'-TCCCATGGATCACAGCCTCT -3'; mouse Ctbp2 F- 5'- GGCAAGGTGCATTCCTTGTG -3', R5'- TCGTATCCTGCCCTCCTTGA -3'; Mouse Lgr5 F- 5'- ACCAGCTTACCCCATGACTG -3', R- 5'CTCCTGCTCTAAGGCACCAC -3'.

\section{Mice}

All animal studies performed for this manuscript were approved by VCU Institutional Animal Care and Use Committee. The C57BL/6J male mice heterozygous for the Apc Min allele ( $\left.A p c^{\mathrm{min} /+}\right)$ and $\mathrm{C} 57 \mathrm{BL} / 6 \mathrm{~J}$ male mice heterozygous for the $C t b p 2$ allele $\left(C t b p 2^{+/}\right)$were purchased from Jackson Laboratory (Bar Harbor, ME). Each genotype was established by mating the founder male with wildtype C57BL/6J female mice with further backcross of the mutant genotypes to $\mathrm{C} 57 \mathrm{BL} / 6 \mathrm{~J}$ at least 6 generations before initiating experiments. Allele-specific PCR assays were used to identify the $A p c$ Min and the $C t b p 2$ mutations (end point PCR: $A p c^{\mathrm{min} /+}$, F- 5'-GGGAAGTTTAGACAGTTCTCGT3'and R-5'-TGTTGGATGGTAAGCACTGAG-3', Mutant5'- AGACAGAAGTTAGGAGAGAGAGC-3'and WT5'-AGACAGAAGTTTGGAGAGAGAGC-3'. Ctbp2 +/- Internal Control, F- 5'-CAAATGTTGCTTGTCTGGTG3'and R- 5'-GTCAGTCGAGTGCACAGTTT-3', Mutant F-5'-CCAGTGGGGATCGACGGTATC-3', Mutant R5'-CACTCCAACGCAGCACCATC-3'). All mice were fed regular water and chow and optimum day and night cycles were maintained.

\section{Mouse small intestinal mucosal cell isolation}

Mouse small intestines were isolated and flushed with HBSS with $2 \%$ glucose solution 2 times in closed conformation. Intestinal slices were cut longitudinally along the length and cleaned for debris and fecal matter. The intestines were cut and chopped into fine pieces of about $2 \mathrm{~mm}$ in size and stored in $\mathrm{HBSS}+2 \%$ glucose on ice. Tissue was resuspended in HBSS-glucose-dispasecollagenase solution and placed on a shaker for $25 \mathrm{~min}$ at $25^{\circ} \mathrm{C}$. The digested tissue was further disaggregated by hand pipetting for $3 \mathrm{~min}$. This was followed by 3 slow centrifugations at $300 \mathrm{rpm}$ with resuspension of pellets in HBSS $+2 \%$ glucose to further purify the intestinal mucosal cell population.

\section{Flow cytometry}

Isolated mouse small intestinal mucosal cells were incubated with conjugated antibody for 30 minutes at $4^{\circ} \mathrm{C}$ and washed with PBS buffer prior to analysis. The following antibodies and dilutions were used: CD133/1 (AC133)APC (1:33 dilution) (Miltenyi Biotec, Auburn, CA, USA); CXCR4-PE conjugated clone 2B11 (Dilution 1:50) (BD Pharmingen, San Jose, CA, USA); CD24- PE conjugated clone M1/69 (BD Pharmingen). Stained cells were run in the Fortessa flow cytometer (BD Biosciences, San Jose, CA, USA) and data were analyzed using FCS Express 4 Flow Research Edition software (De-Novo Software, Glendale, CA, USA) and FACS diva (BD biosciences).

\section{Immunohistochemistry and immunofluorescence}

Mouse intestinal swiss rolls were formalin fixed and paraffin embedded on slides and were subjected to deparaffinization and hydration steps followed by quenching and peroxidase reaction steps [43]. Antigen retrieval was performed using a pressure cooker, followed by blocking for an hour 5\% goat serum and incubation with primary antibodies at a dilution of 1:100 in blocking buffer with CtBP2 (Cat no. 612044, BD Biosciences); CD133 (Cat no. 18470-1-AP, Proteintech, Rosemont, IL, USA); c-Myc (N262, Cat no. sc-764, Santa Cruz Biotechnology, SantaCruz, CA, USA); cyclin D1 (DCS-6, Cat no. 20044, Santa Cruz Biotechnology) overnight. After 3 washes in PBS, secondary antibodies (HRP conjugated anti mouse or anti rabbit IgG, Cat no. K4000, Dako Envision Systems, Santa Clara, CA, USA) were incubated for 1 hour followed by 3 washes in PBS and slide development using DAB chromogen substrate from Dako Envision Systems (Cat no. K3467). Nuclear staining was performed by incubation in hematoxylin for 2 mins and was followed by dehydration and cover-slipping steps. For IF, steps were similar to IHC, except the secondary antibodies used for detection of CD133 and CtBP2 were anti rabbit (Alexa fluor 594; Cat\# A11012; Invitrogen) and anti mouse (Cruz fluor 488; Cat\# sc-362257; Santa Cruz Biotechnology), respectively, followed by similar wash steps as IHC. After dehydration steps, slides were mounted with mounting media containing DAPI (Cat no. S36938, Invitrogen, Eugene, OR, USA) and air dried before analysis.

\section{CRISPR/Cas9-mediated deletion of CtBP2 in HCT116 cells}

Annealed DNA oligonucleotides DP225-5'- TGC AGA CGG GAT GTT GCA CAG TTT T -3'; DP226- 5'TGT GCA ACA TCC CGT CTG CAC GGT G -3') that coded for the target specific crRNa were ligated with linear GeneArt CRISPR Nuclease Vector with Orange Fluorescent Protein (OFP) (Cat No: A21174; Thermo Fisher Scientific, Waltham, MA, USA). Electrocompetent E.coli (DH1OB) were transformed and grown overnight on LB-Agar plates supplemented with $100 \mu \mathrm{g} / \mathrm{ml}$ Ampicillin. Individual clones were purified by streaking and their plasmid DNA was verified by sequencing. HCT116 cells at $70 \%$ confluency, grown in RPMI 1640 supplemented 
with $10 \% \mathrm{FBS}$, in a $100 \mathrm{~mm}$ dish were transfected with $6 \mu \mathrm{g}$ DNA using Lipofectamine 2000. The cells were incubated at $34^{\circ} \mathrm{C}\left(5 \% \mathrm{CO}_{2}\right)$ for 72 hours and OFP positive cells were single cell fluorescence activated cell sorted in a 96 well plate using Aria-BD, (San Jose, CA, USA)

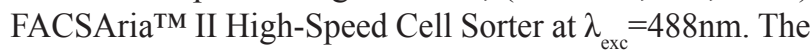
cells were allowed to grow to confluency before splitting them in triplicate plates to screen for mutant clones.

Clones were washed in cold PBS and lysed in 96 well plates using $50 \mu \mathrm{L}$ RIPA buffer supplemented with Protease inhibitor cocktail (Roche). Samples were clarified by centrifugation, prepared for SDS PAGE, and resolved on 15 well Bis-Tris Gradient polyacrylamide gels (4-12\%) (NuPAGE, Thermo Fisher Scientific) using MOPS as a running buffer followed by a wet transfer to a $0.45 \mu \mathrm{m}$ PVDF membrane (Immobilon-FL, CAT. NO: IPFL00010, Millipore). The membranes were incubated overnight with Anti-CtBP2 Mouse mAb (Catalog No.612044 BD Biosciences) and anti-Vinculin (E1E9V) Rabbit mAb (Cell Signalling, Beverly, MA, USA) followed by $1 \mathrm{~h}$ incubation with Alexafluor (680) labelled secondary antibodies (Thermo Fisher Scientific). The blots were scanned at $685 \mathrm{~nm}$ (Odyssey CLx scanner, Odyssey, Lincoln, NE, USA).

After screening, mutant clones were grown to confluency in 6 well plates and genomic DNA was extracted using ISOLATE II Genomic DNA Kit (Cat No. BIO-52067, Bioline, Taunton, MA, USA) as per the manufacturer's instructions. The mutated region of the alleles was PCR amplified using oligonucleotides DP261 and DP262 (DP261-5'- CTG CCA GCT CCA TGA GGG AG -3'; DP262- 5'- CAG GCT GGG GTT GCC TTT CC -3') with Taq Polymerase (Cat No: BIO-25043, MyTaq Red Mix, Bioline), purified using Qiagen PCR purification kit and ligated with pGEM®-T Vector (Cat No: A3600, Promega, Madison, WI, USA). Electrocompetent E.coli (DH1OB) were transformed with the ligation mix and blue-white screening was performed. White colonies were grown overnight; plasmid DNA was extracted and sequenced to confirm mutations.

\section{Abbreviations}

CtBP2: C terminal binding protein 2; $\mathrm{Apc}^{\mathrm{min}}$ : Adenomatous Polyposis Coli Multiple Intestinal Neoplasia; TIC: Tumor initiating cells; FAP: Familial Adenomatous Polyposis; CRISPR: Clustered Regularly Interspaced Short Palindromic Repeats; HIPP: 2-hydroxyimino-3-phenyl-propionic acid; MTOB- $\alpha$-keto$\gamma$-(methylthio) butyric acid.

\section{Author contributions}

ATC, SRG, and BP designed experiments; ATC, ADC and BS performed experiments; ATC, SRG, MI, and BP analyzed data; ATC drafted manuscript; SRG edited manuscript; KCE provided reagents.

\section{ACKNOWLEDGMENTS}

The authors would like to thank members of the Litovchick and Grossman labs for helpful advice.

\section{CONFLICTS OF INTEREST}

The authors have declared that no conflicts of interest exists.

\section{FUNDING}

Services and products in support of this research project were generated by the VCU Massey Cancer Center Flow Cytometry and Cancer Mouse Model Shared Resources, supported, in part, with funding from NIH-NCI Cancer Center Support Grant P30 CA016059. SRG was supported by NCI P30-CA016059 and BP was supported by VA Merit Award 5 I01 BX000837.

\section{REFERENCES}

1. Knudsen AL, Bisgaard ML, Bulow S. Attenuated familial adenomatous polyposis (AFAP). A review of the literature. Fam Cancer. 2003; 2: 43-55.

2. Sumner ET, Chawla AT, Cororaton AD, Koblinski JE, Kovi RC, Love IM, Szomju BB, Korwar S, Ellis KC, Grossman SR. Transforming activity and therapeutic targeting of C-terminal-binding protein 2 in Apc-mutated neoplasia. Oncogene. 2017; 36: 4810-6. https://doi.org/10.1038/ onc.2017.106.

3. Straza MW, Paliwal S, Kovi RC, Rajeshkumar B, Trenh P, Parker D, Whalen GF, Lyle S, Schiffer CA, Grossman SR. Therapeutic targeting of C-terminal binding protein in human cancer. Cell Cycle. 2010; 9: 3764-74. https://doi. org/10.4161/cc.9.18.12936.

4. Deng Y, Deng H, Liu J, Han G, Malkoski S, Liu B, Zhao R, Wang XJ, Zhang Q. Transcriptional down-regulation of Brcal and E-cadherin by CtBP1 in breast cancer. Mol Carcinog. 2012; 51:500-07.

5. Di LJ, Byun JS, Wong MM, Wakano C, Taylor T, Bilke S, Baek S, Hunter K, Yang H, Lee M, Zvosec C, Khramtsova G, Cheng F, et al. Genome-wide profiles of CtBP link metabolism with genome stability and epithelial reprogramming in breast cancer. Nat Commun. 2013; 4:1449.

6. Bizama C, Benavente F, Salvatierra E, Gutiérrez-Moraga A, Espinoza JA, Fernández EA, Roa I, Mazzolini G, Sagredo EA, Gidekel M, Podhajcer OL. The lowabundance transcriptome reveals novel biomarkers, specific intracellular pathways and targetable genes associated with advanced gastric cancer. International Journal of Cancer. 2014; 134: 755-64. https://doi.org/10.1002/ijc.28405.

7. Barroilhet L, Yang J, Hasselblatt K, Paranal RM, Ng SK, Rauh-Hain JA, Welch WR, Bradner JE, Berkowitz RS, 
$\mathrm{Ng}$ SW. C-terminal binding protein-2 regulates response of epithelial ovarian cancer cells to histone deacetylase inhibitors. Oncogene. 2013; 32: 3896-903. https://doi. org/10.1038/onc.2012.380.

8. Wang R, Asangani IA, Chakravarthi BV, Ateeq B, Lonigro RJ, Cao Q, Mani RS, Camacho DF, McGregor N, Schumann TE, Jing X, Menawat R, Tomlins SA, et al. Role of transcriptional corepressor CtBP1 in prostate cancer progression. Neoplasia. 2012; 14:905-14. https:// doi.org/10.1593/neo.121192.

9. Kovi RC, Paliwal S, Pande S, Grossman SR. An ARF/ CtBP2 complex regulates BH3-only gene expression and p53-independent apoptosis. Cell Death Differ. 2010; 17:513-21.

10. Paliwal S, Kovi RC, Nath B, Chen YW, Lewis BC, Grossman SR. The alternative reading frame tumor suppressor antagonizes hypoxia-induced cancer cell migration via interaction with the $\mathrm{COOH}$-terminal binding protein corepressor. Cancer Res. 2007; 67:9322-29. https:// doi.org/10.1158/0008-5472.CAN-07-1743.

11. Grooteclaes M, Deveraux Q, Hildebrand J, Zhang Q, Goodman RH, Frisch SM. C-terminal-binding protein corepresses epithelial and proapoptotic gene expression programs. Proc Natl Acad Sci USA. 2003; 100:4568-73. https://doi.org/10.1073/pnas.0830998100.

12. Zhang Q, Wang SY, Nottke AC, Rocheleau JV, Piston DW, Goodman RH. Redox sensor CtBP mediates hypoxiainduced tumor cell migration. Proc Natl Acad Sci USA. 2006; 103:9029-33.

13. Paliwal S, Ho N, Parker D, Grossman SR. CtBP2 Promotes Human Cancer Cell Migration by Transcriptional Activation of Tiam1. Genes Cancer. 2012; 3: 481-90. https://doi. org/10.1177/1947601912463695.

14. Jin W, Scotto KW, Hait WN, Yang JM. Involvement of CtBP1 in the transcriptional activation of the MDR1 gene in human multidrug resistant cancer cells. Biochem Pharmacol. 2007; 74:851-59.

15. Hahn WC, Weinberg RA. Rules for making human tumor cells. N Engl J Med. 2002; 347:1593-603.

16. Fjeld CC, Birdsong WT, Goodman RH. Differential binding of $\mathrm{NAD}+$ and $\mathrm{NADH}$ allows the transcriptional corepressor carboxyl-terminal binding protein to serve as a metabolic sensor. Proc Natl Acad Sci USA. 2003; 100:9202-07.

17. Mani-Telang P, Sutrias-Grau M, Williams G, Arnosti DN. Role of NAD binding and catalytic residues in the C-terminal binding protein corepressor. FEBS Letters. 2007; 581: 5241-6. https://doi.org/10.1016/j.febslet.2007.10.011.

18. Korwar S, Morris BL, Parikh HI, Coover RA, Doughty TW, Love IM, Hilbert BJ, Royer WE Jr, Kellogg GE, Grossman $\mathrm{SR}$, Ellis KC. Design, synthesis, and biological evaluation of substrate-competitive inhibitors of C-terminal Binding Protein (CtBP). Bioorg Med Chem. 2016; 24:2707-15.

19. Patel J, Baranwal S, Love IM, Patel NJ, Grossman SR, Patel $\mathrm{BB}$. Inhibition of C-terminal binding protein attenuates transcription factor 4 signaling to selectively target colon cancer stem cells. Cell Cycle. 2014; 13: 3506-18. https:// doi.org/10.4161/15384101.2014.958407.

20. Abdul Khalek FJ, Gallicano GI, Mishra L. Colon cancer stem cells. Gastrointest Cancer Res. 2010; (Suppl 1):S16-23.

21. Barker N, Ridgway RA, van Es JH, van de Wetering M, Begthel H, van den Born M, Danenberg E, Clarke AR, Sansom OJ, Clevers H. Crypt stem cells as the cells-oforigin of intestinal cancer. Nature. 2009; 457: 608-11. https://doi.org/10.1038/nature07602.

22. Dick JE. Breast cancer stem cells revealed. Proc Natl Acad Sci U S A. 2003; 100: 3547-9. https://doi.org/10.1073/ pnas.0830967100.

23. Medema JP. Cancer stem cells: the challenges ahead. Nat Cell Biol. 2013; 15: 338-44. https://doi.org/10.1038/ ncb2717.

24. Skoudy A, Hernandez-Munoz I, Navarro P. Pancreatic ductal adenocarcinoma and transcription factors: role of c-Myc. J Gastrointest Cancer. 2011; 42: 76-84. https://doi. org/10.1007/s12029-011-9258-0.

25. Barker N, van Es JH, Kuipers J, Kujala P, van den Born M, Cozijnsen M, Haegebarth A, Korving J, Begthel H, Peters PJ, Clevers H. Identification of stem cells in small intestine and colon by marker gene Lgr5. Nature. 2007; 449: 1003-7. https://doi.org/10.1038/nature06196.

26. Jaggupilli A, Elkord E. Significance of CD44 and CD24 as cancer stem cell markers: an enduring ambiguity. Clin Dev Immunol. 2012; 2012: 708036. https://doi. org/10.1155/2012/708036.

27. Snippert HJ, van Es JH, van den Born M, Begthel $H$, Stange DE, Barker N, Clevers H. Prominin-1/CD133 marks stem cells and early progenitors in mouse small intestine. Gastroenterology. 2009; 136: 2187-94 el. https:/doi. org/10.1053/j.gastro.2009.03.002.

28. Zhu L, Gibson P, Currle DS, Tong Y, Richardson RJ, Bayazitov IT, Poppleton H, Zakharenko S, Ellison DW, Gilbertson RJ. Prominin 1 marks intestinal stem cells that are susceptible to neoplastic transformation. Nature. 2009; 457: 603-7. https://doi.org/10.1038/nature07589.

29. Benovic JL, Marchese A. A new key in breast cancer metastasis. Cancer Cell. 2004; 6: 429-30. https://doi. org/10.1016/j.ccr.2004.10.017.

30. Kucia M, Reca R, Miekus K, Wanzeck J, Wojakowski W, Janowska-Wieczorek A, Ratajczak J, Ratajczak MZ. Trafficking of normal stem cells and metastasis of cancer stem cells involve similar mechanisms: pivotal role of the SDF-1-CXCR4 axis. Stem Cells. 2005; 23: 879-94. https:// doi.org/10.1634/stemcells.2004-0342.

31. Mukherjee D, Zhao J. The Role of chemokine receptor CXCR4 in breast cancer metastasis. Am J Cancer Res. 2013; 3: 46-57.

32. Phillips RJ, Burdick MD, Lutz M, Belperio JA, Keane MP, Strieter RM. The stromal derived factor-1/CXCL12-CXC chemokine receptor 4 biological axis in non-small cell lung 
cancer metastases. Am J Respir Crit Care Med. 2003; 167: 1676-86. https://doi.org/10.1164/rccm.200301-071OC.

33. Margolin DA, Silinsky J, Grimes C, Spencer N, Aycock M, Green H, Cordova J, Davis NK, Driscoll T, Li L. Lymph node stromal cells enhance drug-resistant colon cancer cell tumor formation through SDF-1alpha/CXCR4 paracrine signaling. Neoplasia. 2011; 13: 874-86.

34. Zeelenberg IS, Ruuls-Van Stalle L, Roos E. The chemokine receptor CXCR4 is required for outgrowth of colon carcinoma micrometastases. Cancer Res. 2003; 63: 3833-9.

35. Zhao Y, Peng J, Zhang E, Jiang N, Li J, Zhang Q, Zhang $\mathrm{X}$, Niu Y. CD133 expression may be useful as a prognostic indicator in colorectal cancer, a tool for optimizing therapy and supportive evidence for the cancer stem cell hypothesis: a meta-analysis. Oncotarget. 2016; 7: 10023-36. https://doi. org/10.18632/oncotarget.7054.

36. Chen S, Song X, Chen Z, Li X, Li M, Liu H, Li J. CD133 expression and the prognosis of colorectal cancer: a systematic review and meta-analysis. PLoS One. 2013; 8: e56380. https://doi.org/10.1371/journal.pone.0056380.

37. Hamada F, Bienz M. The APC tumor suppressor binds to $\mathrm{C}$-terminal binding protein to divert nuclear betacatenin from TCF. Dev Cell. 2004; 7: 677-85. https://doi. org/10.1016/j.devcel.2004.08.022.

38. Rennoll S, Yochum G. Regulation of MYC gene expression by aberrant Wnt/beta-catenin signaling in colorectal cancer. World J Biol Chem. 2015; 6: 290-300. https://doi. org/10.4331/wjbc.v6.i4.290.

39. Dcona MM, Morris BL, Ellis KC, Grossman SR. CtBP- an emerging oncogene and novel small molecule drug target: Advances in the understanding of its oncogenic action and identification of therapeutic inhibitors. Cancer Biol Ther. 2017; 18: 379-91. https://doi.org/10.1080/15384047.2017. 1323586 .

40. Chinnadurai G. Transcriptional regulation by C-terminal binding proteins. Int J Biochem Cell Biol. 2007; 39: 1593 607. https://doi.org/10.1016/j.biocel.2007.01.025.

41. Neufeld KL, White RL. Nuclear and cytoplasmic localizations of the adenomatous polyposis coli protein. Proc Natl Acad Sci U S A. 1997; 94: 3034-9.

42. Zhou BB, Zhang H, Damelin M, Geles KG, Grindley JC, Dirks PB. Tumour-initiating cells: challenges and opportunities for anticancer drug discovery. Nat Rev Drug Discov. 2009; 8: 806-23. https://doi.org/10.1038/nrd2137.

43. Shi SR, Liu C, Young L, Taylor C. Development of an optimal antigen retrieval protocol for immunohistochemistry of retinoblastoma protein $(\mathrm{pRB})$ in formalin fixed, paraffin sections based on comparison of different methods. Biotech Histochem. 2007; 82: 301-9. https://doi.org/10.1080/10520290701791763. 